\title{
Blick in die Zukunft
}

\author{
DELPHI-STUDIE Entwicklungen voraussagen hat nichts mit \\ Hokuspokus zu tun, sondern gelingt mit der Delphi-Methode. In \\ mehreren Erhebungsrunden prognostizieren Experten beispiels- \\ weise, welche Trends vor uns liegen.
}

arum der Name eines modernen Erhebungsverfahrens auf das altgriechische Delphi-Orakel zurückgeht, ist ganz einfach: Wie die antike Weissagungsstätte zielt auch die Delphi-Studie darauf ab, unbekannte Themen zu erhellen. Natürlich bedient sie sich dabei keiner übersinnlichen Kräfte; sie nutzt vielmehr die Einschätzung ausgewiesener Experten, um aktuelle Trends oder Zukunftsentwicklungen abzubilden. Dazu nehmen die Experten an einer schriftlichen Befragung teil, die in mehreren Runden stattfindet. Das Besondere daran: Die Experten erhalten nach jeder Runde ein Feedback und können ihre Sichtweisen vergleichen und weiterentwickeln. Damit kann die Methode Gruppenkommunikation steuern und Gruppenmeinungen erfassen [1, 2].

Ihren Namen verdankt die Delphi-Befragung dem Altphilologen Abraham Kaplan. Größere öffentliche Aufmerksamkeit erfuhr sie erstmalig durch die amerikanischen RANDStudien in den 1950er und 1960er Jahren, die militärische Fragen untersuchten [3]. Seit den 1970er Jahren hat sich die Methode im westeuropäischen Raum verbreitet, zum Beispiel in der gesundheitsbezogenen Forschung [1].

Das Problem bestimmt den Zweck > Nicht jede Problemstellung eignet sich für diese Erhebungsmethode. Die meisten DelphiBefragungen untersuchen aktuelle Entwicklungen oder Sachverhalte, die in der Zukunft liegen. Generell bieten sich Themen an, über die bislang nur unvollständiges oder unsicheres Wissen besteht [2]. Dabei legt die gewählte Problemstellung auch fest, welchen Zweck die Studie verfolgen soll. Entsprechend lassen sich vier Typen unterscheiden [1]. Bei Typ 1 geht es darum, möglichst viele Ideen oder Lösungen zu einer Frage zu entwickeln. Typ 2 konzentriert sich darauf, eine in der Zukunft liegende Gegebenheit zu prognostizieren. Mit Typ 3 ermittelt man die Einschätzungen von Experten zu einem ungewissen Sachverhalt. Typ 4 zielt darauf ab, einen Konsens zwischen den Teilnehmern zu erzielen (๑ „Typen“).

Expertenwissen nutzen $>$ In Delphi-Studien geht es nicht um eine reine Wissens- oder Erfahrungsabfrage. Die Urteile beruhen vielmehr auf intuitiven Einschätzungen der Experten, weshalb das Erhebungsverfahren auch zu den „subjektiv-intuitiven Methoden der Vorausschau“ gehört [2].

Dennoch oder gerade deswegen setzt es ein hohes Maß an Expertenwissen voraus. Die Studienteilnehmer müssen die behandelte Problemstellung qualifiziert einschätzen können. Auch sollten sie sich gedanklich in einem abstrakten Raum bewegen und Fragen aus verschiedenen Perspektiven reflektieren können. Die Teilnehmerzahlen von Delphi-Studien variieren erheblich. So finden sich Arbeiten mit nur sieben Experten ebenso wie Befragungen mit weit über 100 Teilnehmern. Dabei gewinnt die Studie nicht zwangsläufig an Aussagekraft, wenn sie eine größere Stichprobe

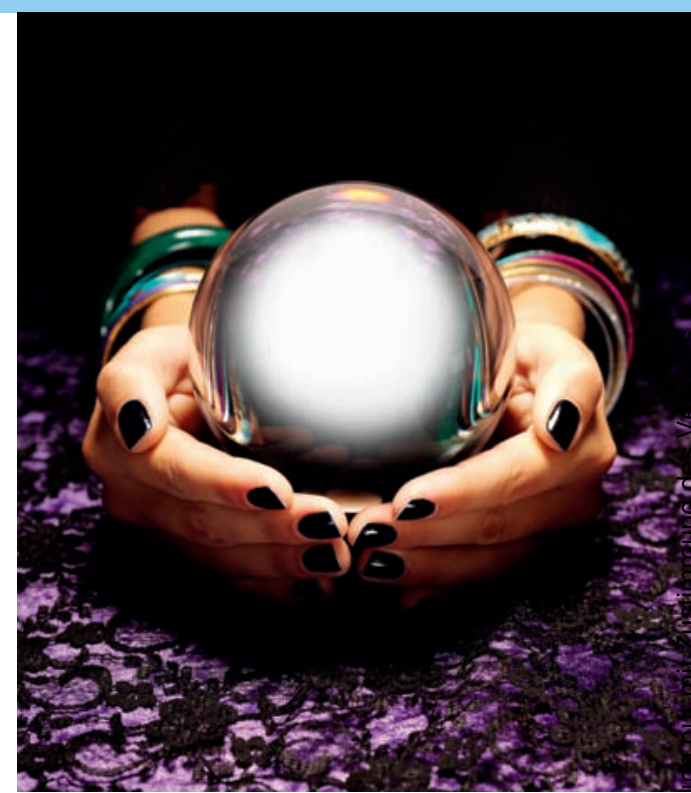

einbezieht. Entscheidend ist vielmehr, dass die Teilnehmer auch tatsächlich über die erforderliche Expertise verfügen [1].

Online-Befragungen sind im Kommen > Obwohl in den ersten Delphi-Studien auch Gruppendiskussionen zum Einsatz kamen, hat sich die schriftliche Befragung etabliert [2]. Charakteristisch dabei ist, dass die Experten unabhängig voneinander an der Studie teilnehmen [4]. Erhielten sie ihre Fragebögen lange Zeit per Post, setzen sich zunehmend auch Online-Befragungen durch.

Normalerweise verläuft die Delphi-Befragung in mindestens zwei Erhebungsrunden: Die Ergebnisse der vorangegangenen Befragung dienen als Grundlage, um den Fragebogen für die nächste Runde zu entwickeln (๑ „Ablauf“). Die Inhalte richten sich neben allgemeinen Empfehlungen vor allem danach, welche Intention die Studie verfolgt. Sollen wie bei einem Konsensverfahren quantitative Übereinstimmungen zwischen den Experten festgestellt werden, dominieren häufig geschlossene Fragen mit vorgegebenen Antwortmöglichkeiten. Geht es darum, Ideen und Einschätzungen zu einem bestimmten Problem zu ermitteln, bieten sich offene Fragen an, die zu qualitativem Datenmaterial führen [1].

Mehrere Runden ermöglichen eine intensive Auseinandersetzung > Nach jeder Erhebungsrunde wertet man die Daten aus und bereitet sie als Grundlage für die fol- 


\section{Typen von Delphi-Studien [1]}

$>$ Typ 1: Ideensammlung zu einem

bestimmten Thema, zum Beispiel welche neuen Arbeitsfelder sich für Ergotherapeuten anbieten

$>$ Typ 2: Vorhersage eines unsicheren Sachverhaltes, beispielsweise welchen gesellschaftlichen Stellenwert die Ergotherapie im Jahre 2030 einnehmen wird

$>$ Typ 3: Expertenansichten zu einem diffusen Sachverhalt wie etwa zu der Frage, welche Rolle die Lebensqualität in der ergotherapeutischen Praxis spielt

$>$ Typ 4: Konsensbildung, bietet sich bei wissenschaftlichen Fachgesellschaften an, die Leitlinien erstellen
Ablauf einer Delphi-Studie [1]

$>$ eine Fragestellung operationalisieren

$>$ einen standardisierten Fragebogen ausarbeiten

$>$ die Befragungsergebnisse aufbereiten

$>$ die Befragung auf Basis der gewonnenen Ergebnisse so lange wiederholen, bis das Abbruchkriterium erreicht ist gende Runde auf. Dieser Prozess wiederholt sich so oft wie vorgesehen oder bis das angestrebte Ziel, zum Beispiel die Konsensfindung, erreicht ist (๑ „Ablauf“).

Gegenüber der einmaligen schriftlichen Befragung bietet die Delphi-Studie einen groBen Vorteil: Die Experten setzen sich vertieft mit einem Problem auseinander. Dabei stimuliert das erhaltene Feedback neue Denkvorgänge und führt zu mentalen Suchprozessen [5]. So können die Teilnehmer ihre Standpunkte und Sichtweisen kontinuierlich weiterentwickeln. Auch die Forscher profitieren davon: Sie lernen die Sichtweisen der Experten während der Datenauswertung kennen und können die Befragungen darauf abstimmen. Allerdings ist der mehrmalige Erhebungs- und Auswertungsprozess auch mit einem hohen zeitlichen, personellen und finanziellen Aufwand verbunden.

\section{Grundlage für Problemlösungen und} Entscheidungen > Sei es, um Trends und Entwicklungen im Gesundheitswesen abzubilden. Oder um zu ermitteln, welche Bedeutung theoretische Konstrukte für die Praxis haben. Längst hat die gesundheitsbezogene Forschung die Delphi-Studie für sich entdeckt. Die skandinavischen Ergotherapeutinnen Ann-Britt Iversson und Maria Müllersdorf untersuchten 2011 zum Beispiel auf diese Weise, wie akademisch ausgebildete Ergotherapeuten den Begriff „occupation“ verstehen [7]. 2009 veröffentlichte das Pharma- unternehmen Janssen Cilag eine Studie, die verschiedene Zukunftsszenarien für das deutsche Gesundheitssystem entwarf und bewertete [6]. Und an der Fachhochschule Bielefeld entstanden in den Jahren 2006 und 2008 zwei Arbeiten zu der Frage, welcher Forschungsbedarf in der Ergo- und Physiotherapie besteht $[8,9]$. Mit Delphi-Befragungen kann man also Problemlösungen entwickeln oder Entscheidungen treffen [3]. Eher ungeeignet ist die Methode, um konkrete Interventionen zu evaluieren oder deren Wirkung zu vergleichen. Außerdem sollte man bedenken, dass sie immer nur die Sichtweisen und Einschätzungen der befragten Experten abbilden kann. Und trotz ausgewiesener Fachkompetenzen gilt natürlich auch für Experten die Devise: „Irren ist menschlich.“

Florence Kranz

$\Rightarrow$ Das Literaturverzeichnis finden Sie unter www.thieme-connect.de/ejournals/toc/ ergopraxis > „Ausgabe 4/12“.

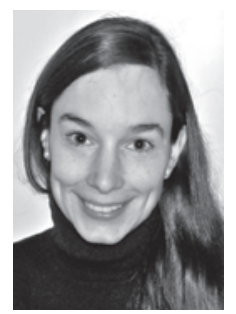

Florence Kranz, Ergotherapeutin BcOT, cand. M.A. Gesundheitsmanagement, begegnet Delphi-Studien, wenn sie Bachelorarbeiten der Hogeschool Zuyd in Heerlen korrigiert oder Studienergebnisse für ergopraxis zusammenfasst. 